\title{
The LysR-type transcriptional regulator Hrg counteracts phagocyte oxidative burst and imparts survival advantage to Salmonella enterica serovar Typhimurium
}

Correspondence

Dipshikha Chakravortty

dipa@mcbl.iisc.ernet.in

Received 14 February 2008

Revised 2 June 2008

Accepted 9 June 2008

\author{
Amit Lahiri, Priyanka Das and Dipshikha Chakravortty
} Centre for Infectious Disease Research and Biosafety Laboratories, Department of Microbiology
and Cell Biology, Indian Institute of Science, Bangalore, India

\begin{abstract}
LysR-type transcriptional regulators (LTTRs) are one of the key players that help bacteria adapt to different environments. We have designated STM0952, a putative LTTR in Salmonella enterica serovar Typhimurium ( $S$. Typhimurium), as hydrogen peroxide resistance gene ( $h r g)$. A hrg knockout mutant of $S$. Typhimurium was sensitive to oxidative products of the respiratory burst, specifically to $\mathrm{H}_{2} \mathrm{O}_{2}$. The hrg mutant is profoundly attenuated in a murine model of infection and showed decreased intracellular proliferation in macrophages. It also induced increased amounts of reactive oxygen species and co-localization with gp91phox in the macrophage cell line, when compared to the wild-type. A strain overexpressing the hrg gene showed a survival advantage over the wild-type Salmonella under $\mathrm{H}_{2} \mathrm{O}_{2}$-induced stress. Microarray analysis suggested the presence of an Hrg regulon, which is required for resistance to the toxic oxidative products of the reticuloendothelial system.
\end{abstract}

\section{INTRODUCTION}

Salmonella enterica comprises a group of Gram-negative bacteria capable of causing clinical syndromes that range from self-limiting diarrhoea to severe fibrinopurulent necrotizing enteritis and life-threatening systemic disease. The survival and replication of $S$. enterica serovar Typhimurium ( $S$. Typhimurium) in macrophages are brought about by regulation of various two-component regulatory systems, transporters and other virulence islands (Garcia Vescovi et al., 1994; Hensel et al., 1995). The mechanisms by which phagocytes kill virulent Salmonella are not completely understood; however, the role of the $\mathrm{NADPH}$ phagocytic oxidase system has been strongly implicated (Mastroeni et al., 2000). The generation of reactive oxygen species (ROS) occurs via a membranebound flavocytochrome $b_{558}$, consisting of two phagocytic oxidase components (gp91phox and p22phox) and four cytosolic components, p40phox, p47phox, p67phox and a GTP-binding Rac protein. During activation, the cytosolic

Abbreviations: $\mathrm{H}_{2}$ DCFDA, 2',7'-dichlorodihydrofluorescein diacetate; LTTR, Lys-type transcriptional regulator; MnTMPyP, Mn(III)tetrakis(1methyl-4-pyridyl)porphyrin pentachloride; PAO, phenyl arsine oxide; ROS, reactive oxygen species.

The GEO accession number for the microarray data for this study is GSE11820.

Supplementary tables with details of strains, vectors and primers, and a spreadsheet of the complete microarray data are available with the online version of this paper. components translocate to the site of gp91phox/p22phox on the phagosomal membrane, forming a functional enzyme complex, which generates ROS by catalysing electron transfer from NADPH to molecular oxygen (Leusen et al., 1994). NADPH phagocytic oxidase (NADPH phox) is active in murine macrophages infected with S. Typhimurium (De Groote et al., 1997), and greater numbers of Salmonella are recovered from the livers and spleens of gp91phox ${ }^{-1-}$ C57BL/6 mice than from wildtype mice (Mastroeni et al., 2000). Further, in the phagocytes, replication of $S$. Typhimurium is limited by NADPH oxidase and MEK/ERK kinase (Rosenberger \& Finlay, 2002). Patients deficient in the NADPH phagocytic oxidase are susceptible to recurrent microbial infection, including salmonellosis (Mouy et al., 1989). To combat the damaging effects of this active oxygen, virtually all aerobic organisms have evolved complex defence and repair mechanisms (Farr \& Kogoma, 1991). For example, the type III secretion system encoded in the SPI2 chromosomal gene cluster of Salmonella has been found to be critical to minimize the contact of Salmonella phagosomes with vesicles harbouring NADPH oxidase or inducible nitric oxide synthase (iNOS) complexes (Vazquez-Torres et al., 2000; Chakravortty et al., 2002).

The LysR family in S. Typhimurium is composed of 44 auto-regulatory LysR-type transcriptional regulators (LTTRs), which apparently evolved from a distant ancestor into subfamilies found in diverse prokaryotic genera (Schell, 1993). In response to different inducers, LTTRs 
activate transcription of linked target genes or unlinked regulons encoding extremely diverse functions including amino acid biosynthesis, nitrogen fixation, oxidative stress response and bacterial virulence (Ochsner et al., 2000; Pagán-Ramos et al., 1998; Russell et al., 2004; Sun et al., 2002). OxyR, a member of the LysR family of bacterial transcriptional factors, regulates expression of many genes required for combating hydrogen peroxide $\left(\mathrm{H}_{2} \mathrm{O}_{2}\right)$ mediated bacterial killing, such as those encoding catalase, glutathione reductase, glutaredoxin, alkyl hydroperoxide reductase, OxyS and Fur (Christman et al., 1985). Twodimensional gel analysis of Salmonella proteins labelled during the $60 \mathrm{~min}$ period following treatment with a low dose of $\mathrm{H}_{2} \mathrm{O}_{2}$ (a ROS) indicated enhanced expression of 30 proteins when compared to untreated cells (Christman et al., 1989).

Microarray data of Eriksson et al. (2003) revealed 8-fold and 10-fold upregulation of two LTTR genes, STM0952 and STM1625, respectively. In this paper, we report that STM0952, but not STM1625, counteracts $\mathrm{H}_{2} \mathrm{O}_{2}$ stress and is implicated in the virulence of $S$. Typhimurium. We hypothesize that STM0952 (which we have named $\mathrm{hrg}$, for hydrogen peroxide resistance gene) can resist oxidantbased killing mechanisms of the host immune system by interfering with ROS production.

\section{METHODS}

Bacterial strains, media and growth conditions. The bacterial strains used in this study (listed in Supplementary Table S1, available with the online version of this paper) are derived from the wild-type (WT) serovar Typhimurium strain 12023 (kindly gifted by Professor M. Hensel). Bacteria were grown at $37{ }^{\circ} \mathrm{C}$ in Luria broth (LB). Carbenicillin, kanamycin and nalidixic acid were used at $50 \mu \mathrm{g} \mathrm{ml}^{-1}$.

Construction of gene deletions in $\mathbf{S}$. Typhimurium. The STM0952 ( hrg) and STM1625 mutants in S. Typhimurium were constructed using a one-step deletion strategy (Datsenko \& Wanner, 2000). Briefly, transformants carrying a 'red' helper plasmid (pKD46) were grown in LB with ampicillin and $10 \mathrm{mM}$ L-arabinose(Sigma) at $30{ }^{\circ} \mathrm{C}$ to an $\mathrm{OD}_{600}$ of $0.35-0.4$ and then made electrocompetent by washing three times with ice-cold $10 \%$ glycerol and MilliQ water. PCR products containing the kanamycin-resistance gene (from plasmid pKD4) flanked by sequences upstream and downstream of the hrg and STM1625 gene were obtained with the sets of primers described in Supplementary Table S2. Electroporation was done according to the manufacturer's instructions (Bio-Rad), using $500 \mathrm{ng}$ PCR product. Transformants were selected on LB agar containing kanamycin. The knockouts were confirmed by PCR using confirmatory primers. In the knockout strains ( $\Delta h r g$ and $\Delta 1625)$ a $1.7 \mathrm{~kb}$ band was amplified, whereas in the WT strain a $1.1 \mathrm{~kb}$ band for both hrg and STM1625 was amplified (data not shown).

Construction of the hrg-complemented (c-hrg) and the hrgoverexpressing strain. DNA extracted from WT $S$. Typhimurium was used as a template to amplify the $\mathrm{hrg}$ gene using primers as listed in Table S1. The amplified product was purified using the Eppendorf Gel Cleanup kit and this insert along with vector pQE-60 were digested with restriction enzymes. The vector and the insert were mixed at $1: 4.5$ molar concentrations and ligated at $16{ }^{\circ} \mathrm{C}$ for $16 \mathrm{~h}$. The vector-containing insert was then transformed into competent cells of Escherichia coli and plated on LB-carbencillin plates after $1 \mathrm{~h}$ incubation in SOC medium (Sambrook et al., 1989). The colonies were screened for the plasmid harbouring the insert, and this purified plasmid was then transformed into $\Delta h r g$ competent cells. The overexpressing strain was constructed similarly (primers in Supplementary Table S1) using the pBAD vector; expression was induced by addition of $1 \mathrm{mM} \mathrm{L}$-arabinose to bacterial cultures $\left(\mathrm{OD}_{600}\right.$ $0.5)$ for $3 \mathrm{~h}$.

Cell culture and bacterial infection. The murine macrophage cell line RAW264.7 was kindly gifted by Professor Anjali Karande (Department of Biochemistry, Indian Institute of Science, India). RAW264.7 and INT 407 cells (obtained from the National Centre for Cell Science, Pune, India) were maintained in Dulbecco's modified Eagle's medium (DMEM; Sigma) containing 10\% heat-inactivated fetal calf serum (Sigma) at $37{ }^{\circ} \mathrm{C}$ in an atmosphere of $5 \% \mathrm{CO}_{2}$. The cells were seeded in 24 -well plates at a density of $2.5 \times 10^{5}$ cells per well for the experiments.

For the infection of RAW264.7 cells, S. Typhimurium strains were grown to stationary phase in LB with the appropriate antibiotic. For infection of epithelial cells, stationary-phase cultures were diluted $1: 33$ in $\mathrm{LB}$ and grown for $3 \mathrm{~h}$. The $\mathrm{OD}_{600}$ of the cultures was adjusted to 0.3 and the resulting bacterial suspensions were used to infect the cells at an m.o.i. of about 10. Bacteria were centrifuged onto the cells at $500 \mathrm{~g}$ for $5 \mathrm{~min}$. After infection for $25 \mathrm{~min}$, cells were washed three times with PBS and incubated for $1 \mathrm{~h}$ in cell culture medium containing $50 \mu \mathrm{g}$ gentamicin $\mathrm{ml}^{-1}$ (Sigma). The medium was replaced with medium containing $10 \mu$ gentamicin $\mathrm{ml}^{-1}$ for the rest of the experiment. In some experiments, IFN $\gamma$ (Sigma, $100 \mathrm{IU} \mathrm{ml}^{-1}$ ), phenylarsine oxide (PAO, $5 \mu \mathrm{M}$ ) or Mn(III)tetrakis(1-methyl-4-pyridyl)porphyrin pentachloride (MnTMPyP; Calbiochem, $50 \mu \mathrm{M}, 30 \mathrm{~min}$ pretreatment) was added to the cells along with $10 \mu \mathrm{g}$ gentamicin $\mathrm{ml}^{-1}$.

For the enumeration of intracellular bacteria, macrophages were washed three times with PBS and lysed with $0.1 \%$ Triton X-100 for $10 \mathrm{~min}$ at room temperature and serial dilutions were plated onto LB agar.

$\mathbf{H}_{\mathbf{2}} \mathbf{O}_{\mathbf{2}}$ sensitivity assay. Cells ( $10^{7}$ c.f.u.) from early stationary-phase cultures ( $9 \mathrm{~h}$ growth) of the WT, $\Delta h r g$ and (L-arabinose-induced) Hrg-overexpressing strains were suspended separately in $1 \mathrm{ml}$ PBS containing $600 \mu \mathrm{M} \mathrm{H}_{2} \mathrm{O}_{2}$. The cultures were grown aerobically with shaking at 225 r.p.m. at $37{ }^{\circ} \mathrm{C}$ and after every time point $1000 \mathrm{U}$ catalase (Sigma) was added to quench the remaining $\mathrm{H}_{2} \mathrm{O}_{2}$. Serial dilutions of the bacteria were plated every hour to monitor c.f.u.

Measurement of extracellular $\mathbf{H}_{\mathbf{2}} \mathrm{O}_{\mathbf{2}}$ by phenol red assay. $\mathrm{H}_{2} \mathrm{O}_{2}$ production by RAW264.7 cells was determined by a modification of the method of Wautier et al. (2001), which uses the horseradish peroxidase (HRPO)-dependent conversion of phenol red by $\mathrm{H}_{2} \mathrm{O}_{2}$ into a compound with increased absorbance at $610 \mathrm{~nm}$. Briefly, RAW264.7 cells after infection with the WT or the $\Delta h r g$ strain were incubated in phenol red solution containing $\mathrm{NaCl}(0.14 \mathrm{M})$, potassium phosphate $(0.01 \mathrm{M} ; \mathrm{pH} 7.0)$, glucose $(0.0055 \mathrm{M})$, phenol red $\left(0.2 \mathrm{~g} \mathrm{l}^{-1}\right)$ and HRPO $\left(19 \mathrm{U} \mathrm{ml}^{-1}\right.$; Sigma). At the end of the incubation period, the supernatant was assayed in a spectrophotometer at $610 \mathrm{~nm}$ to check the amount of $\mathrm{H}_{2} \mathrm{O}_{2}$. From each infected well total protein was estimated using the Bradford method and the data are represented as amount of $\mathrm{H}_{2} \mathrm{O}_{2}$ produced $\mathrm{h}^{-1}$ (mg total protein $)^{-1}$. A standard curve was generated using $\mathrm{H}_{2} \mathrm{O}_{2}$ concentrations ranging from 0.1 to $5 \mu \mathrm{M}$ in the phenol red solution.

Measurement of intracellular ROS. Intracellular ROS level was measured by flow cytometry (Von Knethen \& Brüne, 2001) by using the redox-sensitive dye $2^{\prime}, 7^{\prime}$-dichlorodihydrofluorescein diacetate ( $\mathrm{H}_{2}$ DCFDA; Molecular Probes). $\mathrm{H}_{2}$ DCFDA is a cell-permeable indicator for ROS that is non-fluorescent until the acetate groups 
are removed by intracellular esterases and oxidation to the highly fluorescent polar derivative $\mathrm{H}_{2} \mathrm{DCF}$ occurs within the cell. For this assay, $10^{6}$ cells were incubated for 30 min with $10 \mu \mathrm{M} \mathrm{H}_{2}$ DCFDA in the dark. Cells were quickly washed and immediately 10000 events were acquired on a FACSCalibur (BD Biosciences).

Localization of NADPH subunit gp91phox by fluorescence microscopy. Localization of gp91phox in RAW264.7 cells infected with the WT or $\Delta h r g \mathrm{~S}$. Typhimurium was determined by standard immunocytochemical methods. The WT- or $\Delta h r g$-infected cells were washed free of medium,were fixed for $10 \mathrm{~min}$ in paraformaldehyde $(3.5 \%)$ at room temperature and washed with PBS. Fixed cells were then incubated with goat anti-mouse gp91phox immunoglobulin G (BD Transduction) diluted 1:100 in PBS containing 2\% BSA, 2\% goat serum and $0.1 \%$ saponin. The cells were then washed three times in PBS and incubated in identical conditions but with rabbit anti-goat IgG conjugated to Cy3 (Jackson ImmunoResearch Laboratories). Bacteria were stained with anti-LPS antibody raised in mouse followed by FITC-conjugated anti-mouse IgG. Samples were viewed on a confocal laser-scanning microscope equipped with an argon laser (Zeiss).

Mouse experiments. Six- to eight-week-old BALB/c mice (Central Animal Facility, Indian Institute of Science, Bangalore, India) were maintained under specific-pathogen-free conditions. All procedures with animals were carried out in accordance with institutionally approved protocols. Bacterial strains were grown with shaking overnight at $37^{\circ} \mathrm{C}$, centrifuged, washed, resuspended to the appropriate concentration in sterile PBS, and administered to mice at the indicated doses. For the survival studies, 10 mice were used for each strain of bacteria and observed daily for survival. For the organ infiltration experiments, liver, spleen and mesenteric lymph nodes were taken aseptically 5 days after infection. The organs were weighed and homogenized in $1 \mathrm{ml}$ PBS. The homogenate was centrifuged and plated at different dilutions to determine bacterial numbers.

Competitive index assay. The competitive indices (Beuzon \& Holden, 2001) were calculated by oral infection with the WT and $\Delta h r g$ or WT and c-hrg strains at 1:1 ratio, i.e. organisms of two strains, in three independent experiments. After the 5th day of infection, homogenized samples of liver, spleen and mesenteric lymph nodes of infected mice were plated and the competitive index for the knockout bacteria was calculated by dividing the number of the mutant bacteria from the infected animal by the number of theWT bacteria recovered. This value was then corrected by the initial ratio of the mutant to WT bacteria used to infect the mice. For control experiments, the competitive index of a different isogenic WT strain was calculated in the same way.

Microarray analysis. The microarray analysis of the gene expression profile comparing the WT and the $\Delta h r g$ strain was performed according to the manufacturer's protocol (Genotypic Technology, Agilent Technologies). Total RNA was isolated following treatment with $600 \mu \mathrm{M} \mathrm{H}_{2} \mathrm{O}_{2}$ for $4 \mathrm{~h}$. The hybridization procedure for the total RNA and the complete normalized data with ratio and fold change are available as supplementary data with the online version of this paper. In the knockout strain, the genes that were upregulated more than 1.8-fold and those downregulated more than 0.55 -fold were considered to be significant. The microarray was repeated for these genes and a $P$-value $<0.05$ for any gene was considered significant. The complete dataset has been submitted to the GEO public database (accession no. GSE11820).

Statistical analysis and software. Each assay was repeated at least three times. In vitro data were analysed by paired $t$ test (two sample, equal variance) and $P$ values below 0.05 were considered significant. FACS data were plotted and analysed using WinMDI software.
Results of mouse in vivo challenge studies were evaluated by using the Mann-Whitney $U$ test from the GraphPad Prism 4.0 software. Differences between experimental groups were considered significant at $P<0.05$.

\section{RESULTS}

\section{Hrg is homologous to members of the LTTR family}

In silico analysis revealed that the sequence from amino acid 7 to 70 from the $\mathrm{N}$-terminus of the $\mathrm{Hrg}$ protein corresponds to the HTH motif and the sequence from amino acid 90 to 298 corresponds to the substrate-binding domain. A bioinformatic search against non-redundant protein databases revealed several bacterial proteins that share significant homology with Hrg. All of them are known LTTRs from different bacteria. More precise comparison of $\mathrm{Hrg}$ with these proteins revealed that the sequence similarity is concentrated in the DNA-binding HTH motif and in the substrate-binding domain. Fig. 1 shows the analysis with five known LTTRs from different bacteria; the homology of $\mathrm{Hrg}$ with these proteins is around $65 \%$, suggesting that Hrg is indeed a member of the LysR family of proteins.

\section{The hrg mutant is attenuated for growth in macrophages}

The role of the $\mathrm{hrg}$ gene in intracellular survival was evaluated in RAW264.7, a murine macrophage cell line. As shown in Fig. 2(a), the fold increase in the WT strain from 2 to $16 \mathrm{~h}$ is 10.7 -fold, whereas in the $\Delta h r g$ strain, it is only 2.7 -fold. This decrease in the fold replication was not seen in the hrg-complemented strain. However, a knockout mutant of another LTTR, STM1625, which was also found to be upregulated in the macrophages, replicated like the WT strain when macrophages were infected with this strain $(\Delta 1625)$. This defect in the intracellular proliferation of the $\Delta \mathrm{hrg}$ strain was restricted to macrophage cells and was not observed in INT 407, an intestinal epithelial cell line (Fig. $2 \mathrm{~b})$. The growth of the WT and the $\Delta h r g$ strains was comparable in LB and in minimal media (data not shown).

\section{The hrg mutant is sensitive specifically to $\mathrm{H}_{2} \mathrm{O}_{2}$ and not to other oxidative or nitrosative stresses}

As the $\Delta h r g$ strain showed decreased replication in macrophages compared to the WT strain, we sought to investigate the mechanism behind this attenuation. The WT, $\Delta h r g, c-h r g$ and $\Delta 1625$ strains were therefore subjected to various oxidative and nitrosative stresses. No difference in growth was observed among the strains when methylene blue $\left(0.5 \mathrm{mg} \mathrm{ml}^{-1}\right)$, sodium hypochlorite $\left(0.2 \mathrm{mg} \mathrm{ml}^{-1}\right)$ or acidified sodium nitrite $(1 \mathrm{mM}, \mathrm{pH} 5)$ was used to confer stress (data not shown). However, when the cells were treated with $600 \mu \mathrm{M} \mathrm{H}_{2} \mathrm{O}_{2}$, starting from the fourth hour of treatment the $\Delta h r g$ strain exhibited greater sensitivity to $\mathrm{H}_{2} \mathrm{O}_{2}$ than the WT and $\Delta 1625$, whereas the 


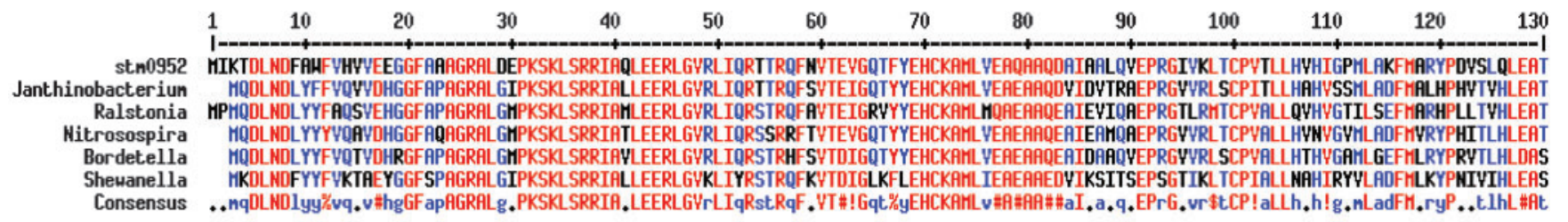

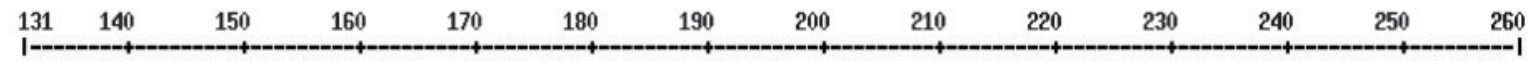

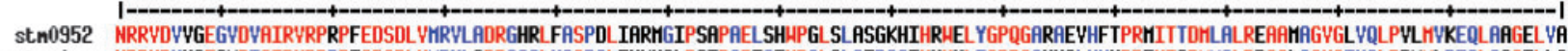

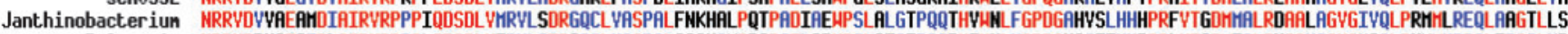

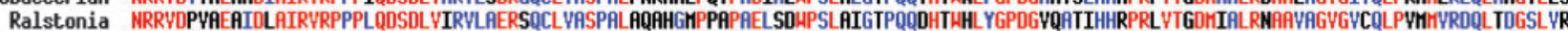

Nitrosospira NRRYDYLSEAYDYAIRYRPPPLODSDLYHRYLADRGOCLYGSOALYHFFGFPRTPGELSNLPSLGLGTPOOIHKLYLHGPNGAOATLHHHPRLITTDHITLRNARLAGYGHYOLPYLIYRDHLARGSLYR

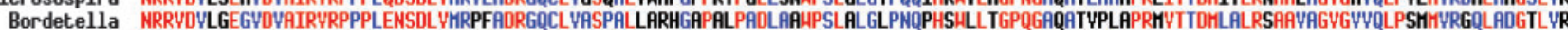

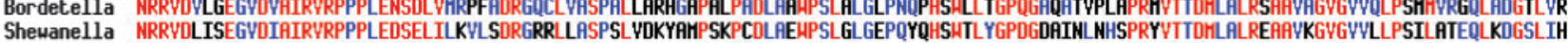

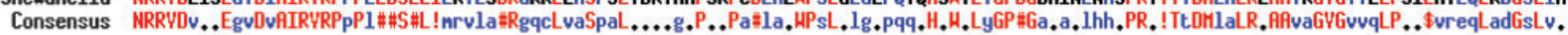

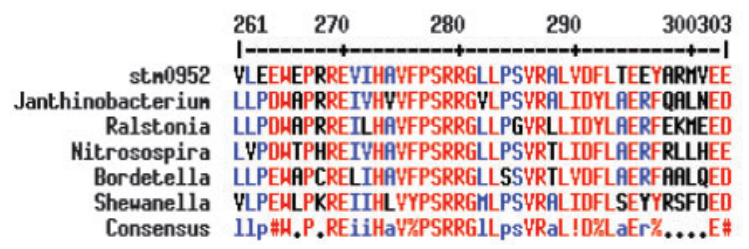

Fig. 1. Hrg is a member of the LTTR family. Amino acid sequence alignment of Hrg with known LTTRs from Janthinobacterium marseille (ABR 90112), Ralstonia metallidurans (YP_586396), Nitrospira multiformis (ABB 74775), Bordetella bronchiseptica (NP_889364) and Shewanella dentrificans (ABE 57020) is presented. Sequence alignment was performed by CLUSTAL W and the output was reformatted by Multalin version 5.4.1. Amino acid identity is shown in red and similar amino acids are shown in blue. The consensus sequence is shown: ! is any one of IV, $\$$ is any one of $L M, \%$ is any one of FY, \# is any one of NDQEBZ.

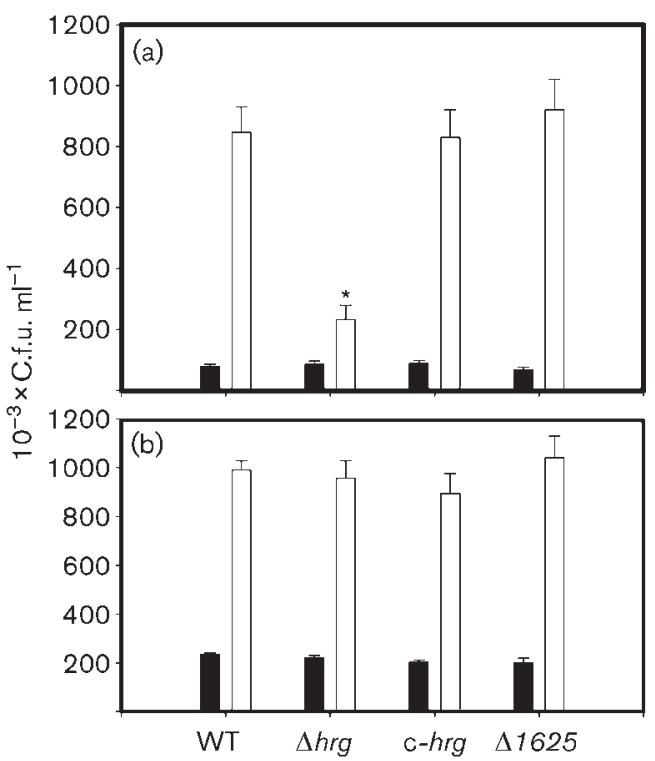

Fig. 2. Disruption of the hrg gene of $S$. Typhimurium hampers intracellular replication in RAW264.7 macrophages (a) but not in INT 407 epithelial cells (b). Infected macrophages and epithelial cells were lysed at $2 \mathrm{~h}$ (black bars) and $16 \mathrm{~h}$ (white bars) post-infection and the bacterial loads were determined. WT represents wild-type $S$. Typhimurium, $\Delta h r g$ harbours a mutation in the STM0952 gene, c- $h r g$ represents complemention of the hrg gene in trans, $\Delta 1625$ represents deletion of the STM1625 gene. Graphs are representative of three independent experiments, each with triplicate samples. Means \pm SEM are shown. ${ }^{*} P<0.05$ (Student's $t$ test).
Hrg-overproducing strain showed increased survival advantage over the WT (Fig. 3b). Overexpression of the Hrg protein in the overexpressing strain was confirmed by SDS-PAGE (Fig. 3a).

\section{RAW264.7 macrophages show increased $\mathrm{H}_{2} \mathrm{O}_{2}$ accumulation in the supernatant and increased intracellular ROS production after infection with the $\Delta$ hrg strain compared to infection with the WT strain}

We measured the amount of $\mathrm{H}_{2} \mathrm{O}_{2}$ produced in the supernatant of infected RAW264.7 cells. The macrophages infected with the WT and the $\Delta h r g$ strain showed a significant difference in the phenol red assay: after $8 \mathrm{~h}$ of infection, the $\Delta h r g$-infected macrophages produced $186.3 \pm 4 \mathrm{nmol} \mathrm{H}_{2} \mathrm{O}_{2} \mathrm{~h}^{-1}$ (mg protein) ${ }^{-1}$ compared to $107.7 \pm 5 \mathrm{nmol} \mathrm{H}_{2} \mathrm{O}_{2} \mathrm{~h}^{-1}$ (mg protein) $)^{-1}$ in the case of the WT infection.

We then tested ROS production in the macrophages after Salmonella infection using the dye $\mathrm{H}_{2}$ DCFDA, which is converted to its fluorescent derivative in the presence of ROS. Only $5.8 \%$ of the macrophages produced ROS upon infection with the WT strain, whereas $15 \%$ of the cells produced ROS when infected with the $\Delta h r g$ strain (Fig. 3c). In INT 407 cells, where no compromise was observed in the intracellular proliferation of the $\Delta h r g$ strain, ROS production was similar in both the WT- and the $\Delta h r g$ infected cells (data not shown). In all cases, uninfected cells served as control. 


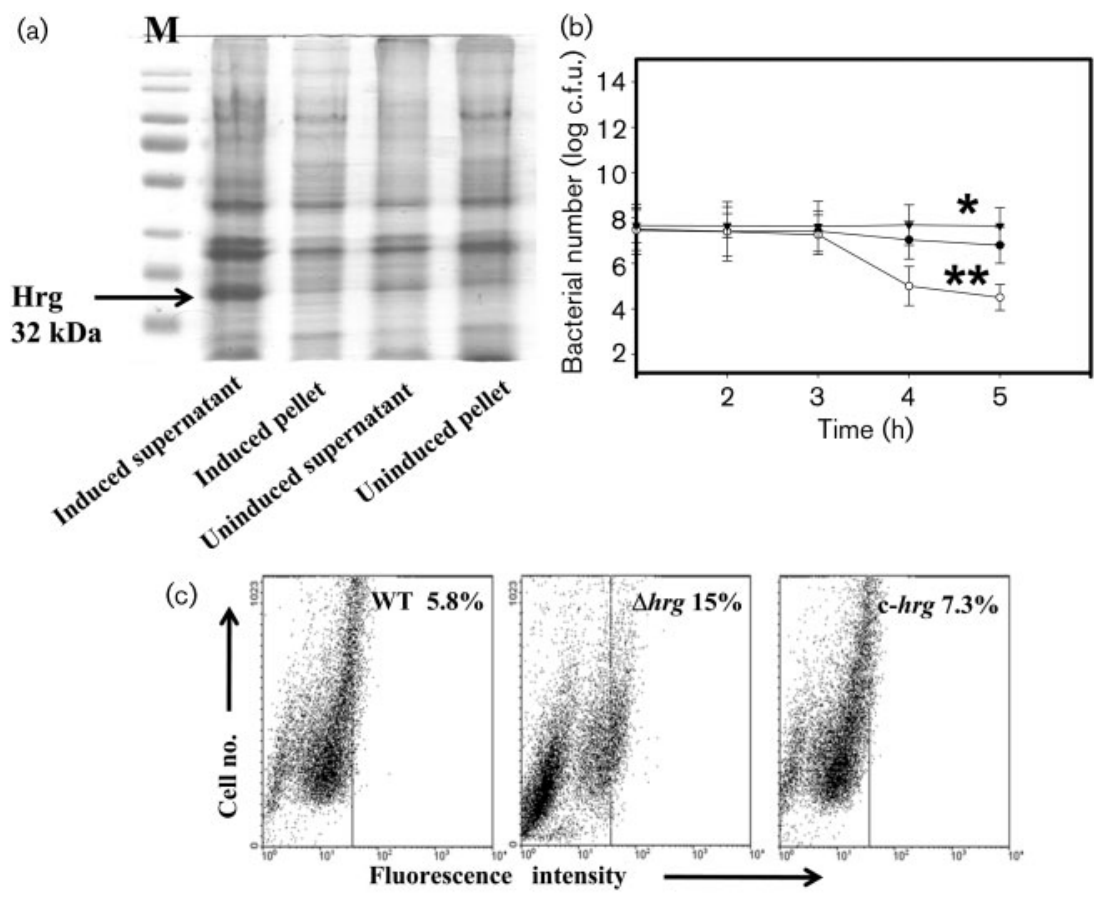

Fig. 3. In vitro and in vivo (cell culture) sensitivity of the WT and $\Delta h r g$ strains to oxidative stress. (a) Induction of Hrg protein from the $P_{B A D}$ promoter in $S$. Typhimurium. Induced cultures were sonicated and centrifuged to obtain the supernatant and pellet fraction. The arrow indicates the Hrg protein. (b) Early stationary-phase cultures of the WT (๑), $\Delta h r g(\bigcirc)$ and overexpressing ( $h r g \mathrm{P}_{\mathrm{BAD}}$, ק) strains were incubated with $600 \mu \mathrm{M} \mathrm{H}_{2} \mathrm{O}_{2}$ for the time period indicated and viable cells were determined by serial dilution and plating on LB agar. (c) ROS production by the WT, $\Delta h r g$ and c-hrg strains in RAW264.7 macrophages. RAW264.7 cells were infected with bacteria for $12 \mathrm{~h}$. The infected or uninfected cells were then incubated with $10 \mu \mathrm{M}$ DCFDA and analysed by FACS for ROS production. Uninfected cells served as a control. Results in (b) and (c) are representative of three independent experiments, each with triplicate samples. Means \pm SEM are shown. ${ }^{*} P<0.05$, ${ }^{* *} P<0.01$ (Student's $t$ test).

\section{Inhibition of ROS production by MnTMPyP or PAO reverses the growth attenuation phenotype of the $\Delta h r g$ strain}

We next checked the fate of the $\Delta h r g$ strain when ROS production was induced in macrophages with IFN $\gamma$. IFN $\gamma$ $\left(100 \mathrm{IU} \mathrm{ml}^{-1}\right.$ ) induced a high level of ROS as assessed by DCFDA staining (data not shown). As shown in Fig. 4(a), addition of IFN $\gamma$ led to a 4 -fold decrease in the intracellular proliferation of the WT and a 10-fold decrease in the case of the $\Delta h r g$ strain.

We sought to determine whether the high level of ROS in the macrophages infected with the $\Delta h r g$ strain contributes to the growth defect. MnTMPyP was used to quench the ROS produced in the cells. As depicted in Fig. 4(b), pretreatment of macrophages with MnTMPyP completely restored the growth of the $\Delta h r g$ strain to the WT level. $\mathrm{PAO}$ is a pharmacological inhibitor of NADPH oxidase. Incubation of macrophages with $\mathrm{PAO}$ gave a similar result to MnTMPyP (Fig. 4c).

\section{Increased co-localization of gp91phox with the $\Delta h r g$ strain}

As it was evident that ROS production was significantly higher in macrophages infected with the $\Delta h r g$ strain than in those infected with the WT, we next examined whether there is any difference in the NADPH phagocytic oxidase localization between the WT and $\Delta h r g$ strain. After infecting RAW264.7 macrophages with either the WT or $\Delta h r g$ for $20 \mathrm{~h}$, cells were stained for Salmonella LPS and gp91phox subunit of NADPH phagocytic oxidase. Interestingly, increased co-localization of the $\Delta \mathrm{hrg}$ mutant and gp91phox was observed as compared to the WT bacteria (Fig. 5). The percentage co-localization of gp91phox with the $\Delta h r g$ strain was $24 \%( \pm 1.7)$ versus $9 \%( \pm 0.71)$ with the WT Salmonella.

\section{Reduced mortality in mice infected with the $\Delta h r g$ strain}

Having demonstrated that $h r g$ is necessary for the replication of $S$. Typhimurium in macrophages, we next investigated the virulence of the $\Delta h r g$ strain in a murine model of typhoid fever. To address this, BALB/c mice $(n=10)$ were orally infected with $10^{7}$ c.f.u. of the WT or the $\Delta h r g$ strain. As shown in Fig. 6(a), mice infected with the WT or c- $h r g$ strain died within 7 days of infection, whereas mice infected with the $\Delta h r g$ strain showed up to $60 \%$ survival until day 7 .

\section{Lower burden of the $\Delta h r g$ strain in the organs of mice}

To investigate whether the reduced mortality of the $\Delta h r g-$ infected mice was due to reduced numbers of bacteria in the organs, we analysed the bacterial load in the spleen, liver and mesenteric lymph nodes after infecting mice orally at a dose of $10^{6}$ bacteria per mouse. After 5 days of infection, there was a significant decrease in the bacterial burden in all the organs of mice infected with the $\Delta h r g$ strain when compared to the mice infected with the WT strain (Fig. 6b-d).

In order to further evaluate the loss in relative virulence displayed by the $\Delta h r g$ strain, we tested the ability of this 
(a)

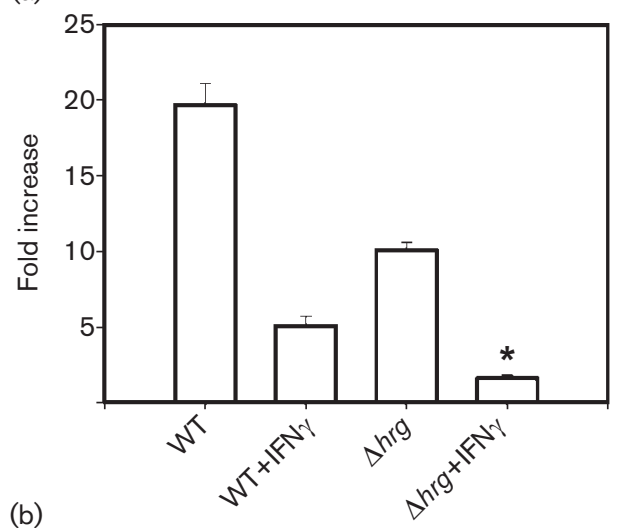

(b)

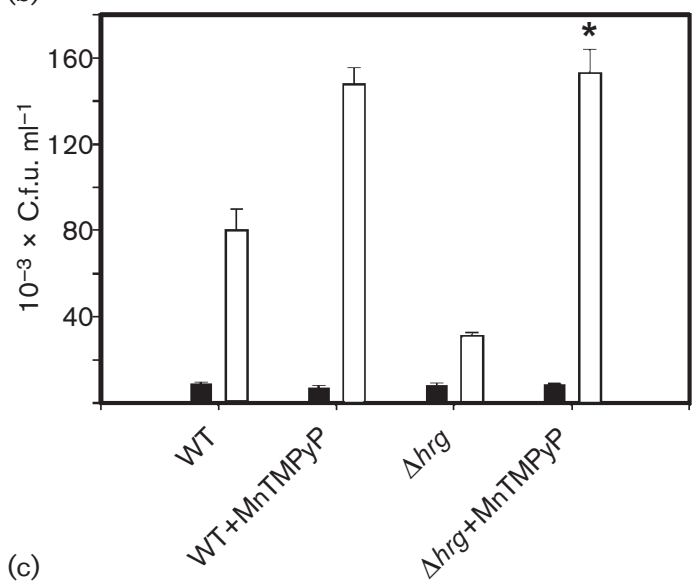

(c)

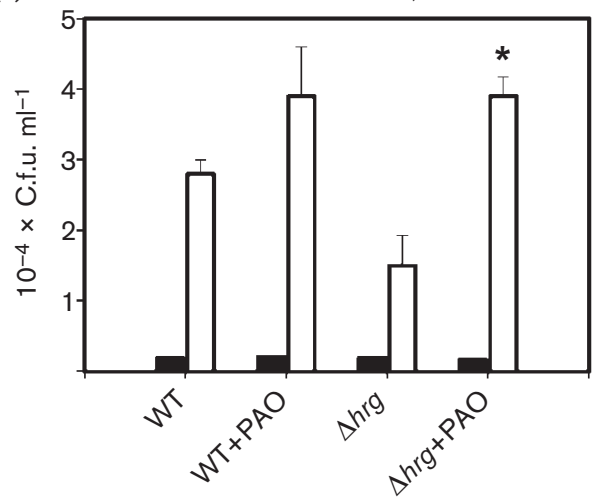

knockout to compete with the WT strain after oral infection. As shown in Table 1 , the $\Delta h r g$ strain exhibited a competitive index defect after the 5 th day of infection in liver, spleen and mesenteric lymph nodes, suggesting that this gene was essential for the in vivo colonization of $S$. Typhimurium. The $P$ values for all the organs were less than 0.05 . The c-hrg strain was found to compete equally against the wild-type strain.

\section{Presence of an Hrg regulon}

Presence of an Hrg regulon was evident from the microarray data (see supplementary data). In the presence of $600 \mu \mathrm{M} \mathrm{H}_{2} \mathrm{O}_{2}$, catalase hydroperoxidase HPI (katG), uvrA and DNA polymerase III theta subunit (holE) were downregulated significantly in the knockout strain when compared to the WT control (Table 2). Acid-shock protein was also downregulated in the $\Delta h r g$ strain. We speculate that these genes might be regulated by $\mathrm{Hrg}$. NADH dehydrogenase genes, several permeases, putative transferases and transporters were also downregulated in the $\Delta h r g$ strain (supplementary data). We also observed the upregulation of several genes required for invasion and transport function in the knockout strain under oxidative stress conditions. However, we restricted our discussion only to the genes that could account for the observed phenotype of the mutant under oxidative stress. The fold difference and the corresponding $P$ values for the genes are summarized in Table 2.

\section{DISCUSSION}

S. Typhimurium is a fascinating pathogen with a wide array of survival strategies against various host defence mechanisms. Pathogenic microbes have developed strategies to resist the antimicrobial effects of NADPH phagocytic oxidase, including the production of molecular scavengers, antioxidant enzymes, repair systems and expression of specific antioxidant regulons (Miller \& Britigan, 1997). For example, the OxyR and SoxRS regulons enable $E$. coli to resist the effects of $\mathrm{H}_{2} \mathrm{O}_{2}$ and superoxide, respectively (Christman et al., 1989; Wu \& Weiss, 1991). However, it has been convincingly shown that $S$. Typhimurium does not require a functional OxyR or SoxRS regulon for virulence (Miller et al., 1989; Taylor et al., 1998), suggesting that this pathogen may use alternative strategies to avoid exposure to high concentrations of phagocyte-derived oxidants in vivo and there might be other regulators that work independently of or in conjunction with OxyR or SoxRS.

The LTTR family plays an important role in oxidative stress response in many pathogenic organisms. Mutational studies and amino acid sequence similarities of LTTRs identify a DNA-binding domain employing a helix-turnhelix motif domain involved in coinducer recognition and/ or response and a domain required for both DNA binding and coinducer response (Schell, 1993). Upon induction by 

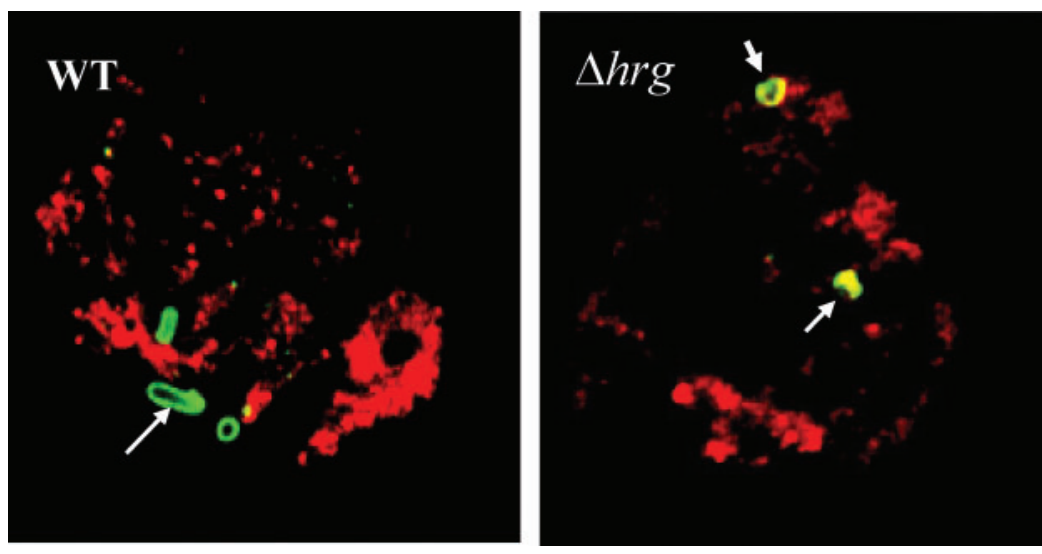

Fig. 5. Localization of NADPH subunit gp91phox with WT and $\Delta h r g$ S. Typhimurium by confocal microscopy. RAW264.7 cells were infected with the WT or $\Delta h r g$ strain for $12 \mathrm{~h}$ and stained with goat anti-mouse gp91phox IgG rabbit antibody followed by anti-goat IgG antibody conjugated to Cy3 (red); bacteria were stained with anti-LPS antibody followed by FITC-conjugated antimouse IgG antibody (green). Representative pictures are shown.

the coinducers, the LTTRs can regulate diverse genes and their functions. OxyR, a LTTR, upregulates various genes upon $\mathrm{H}_{2} \mathrm{O}_{2}$ treatment in different bacteria (Farr \& Kogoma, 1991) In oxidized form it binds to the promoter of the genes it regulates. There has been one report suggesting that OxyR can act as a repressor of catalase expression in Neisseria gonorrhoeae (Tseng et al., 2003). In Salmonella, another LTTR, SpvR, has been studied extensively and has been found to be important for spleen invasion; it is expressed only during the stationary phase of bacterial growth (Libby et al., 1997). The mechanism of the $s p v$ effect is via induction of cytopathology in the host macrophages (Libby et al., 2000). The $s p v$ virulence locus, although it is found on large plasmids in Salmonella subspecies I serovars, is located on the chromosome of serovar Arizona (Libby et al., 2002). It has also been demonstrated that polynucleotide phosphorylase of Salmonella negatively controls $s p v$ virulence gene expression (Ygberg et al., 2006). Various other LTTRs in S. Typhimurium take part in biosynthesis of amino acids such as cysteine, methionine and lysine (Kredich, 1971).

\section{Hrg imparts survival advantage to $S$. Typhimurium and is a virulence factor}

The data presented here show an important role of the $\mathrm{hrg}$ (STM0952) gene in S. Typhimurium pathogenesis and resistance to the antimicrobial activity of macrophages. In spite of its upregulation in the macrophage, the role of this gene previously remained uncharacterized. In silico analysis predicts that STM0952 is a member of the LTTR family, as it has significant homology with other known LysR family proteins from different bacteria. We named STM0952 as $h r g$, for 'hydrogen peroxide resistance gene'. We report here that the $h r g$ gene confers resistance of $S$. Typhimurium to oxidative stress, specifically to $\mathrm{H}_{2} \mathrm{O}_{2}$, and is important for survival in the macrophage population. We demonstrate that a $h r g$ mutant has reduced capacity to survive and replicate in RAW264.7 macrophages. We have also shown that although the hrg mutant is able to reach extra-intestinal sites, namely, the liver, spleen and mesenteric lymph nodes, to invade deep tissues, it is unable to proliferate there efficiently. The $\Delta \mathrm{hrg}$ strain also showed a competitive index defect in liver, spleen and mesenteric lymph nodes, indicating that the attenuation of this strain diminishes its ability to compete for survival in these organs. This suggests that $S$. Typhimurium is unable to withstand host cellular antimicrobial mechanisms in the absence of the Hrg-regulated genes.

\section{Genes necessary to counteract $\mathrm{H}_{2} \mathrm{O}_{2}$ stress are non-functional in the $\Delta h r g$ strain}

The intracellular growth defect of the $\Delta h r g$ but not the $\Delta 1625$ mutant in macrophages further suggested that these two LTTRs might have different functions. The fact that the defect was found in macrophages but not in epithelial cells could indicate that the attenuation of the knockout strain is be due to increased oxidative burst. The elicitation of phagocyte oxidative burst after pathogen entry is well documented (Wilson et al., 1980; Wojtaszek, 1997). Reactive oxygen radicals are one of the potent antimicrobial responses that phagocytes use to limit the growth of micro-organisms. $\mathrm{H}_{2} \mathrm{O}_{2}$ is a key component of the oxidative burst of host macrophages. In the bacterial cell $\mathrm{H}_{2} \mathrm{O}_{2}$ can react with iron or copper ions to generate the toxic hydroxyl radical $\left(\mathrm{OH}^{\circ}\right)$ via the Fenton reaction (Cadenas, 1989). To survive and thrive, intracellular pathogens have to either avoid elicitation of oxygen radicals or resist their effects. After infection with the WT S. Typhimurium, 5\% of the total macrophage population was positive for ROS production. This result is in accordance with data of Foster et al. (2003), who reported that WT S. Typhimurium induced very low levels of intracellular ROS as compared to the phoP mutant. We found a similar result in the phenol red assay. Macrophages infected with the $\Delta h r g$ strain showed increased $\mathrm{H}_{2} \mathrm{O}_{2}$ production in the supernatant compared to those infected with the wild-type. The higher level of intracellular ROS production seen in infections with the $\Delta h r g$ strain might be due to its inability to quench the $\mathrm{H}_{2} \mathrm{O}_{2}$ produced, thereby leading to increased ROS accumulation. MnTMPyP is a potent ROS quencher and mimics catalase (Xiao et al., 2002). MnTMPyP pretreatment of the host macrophages 

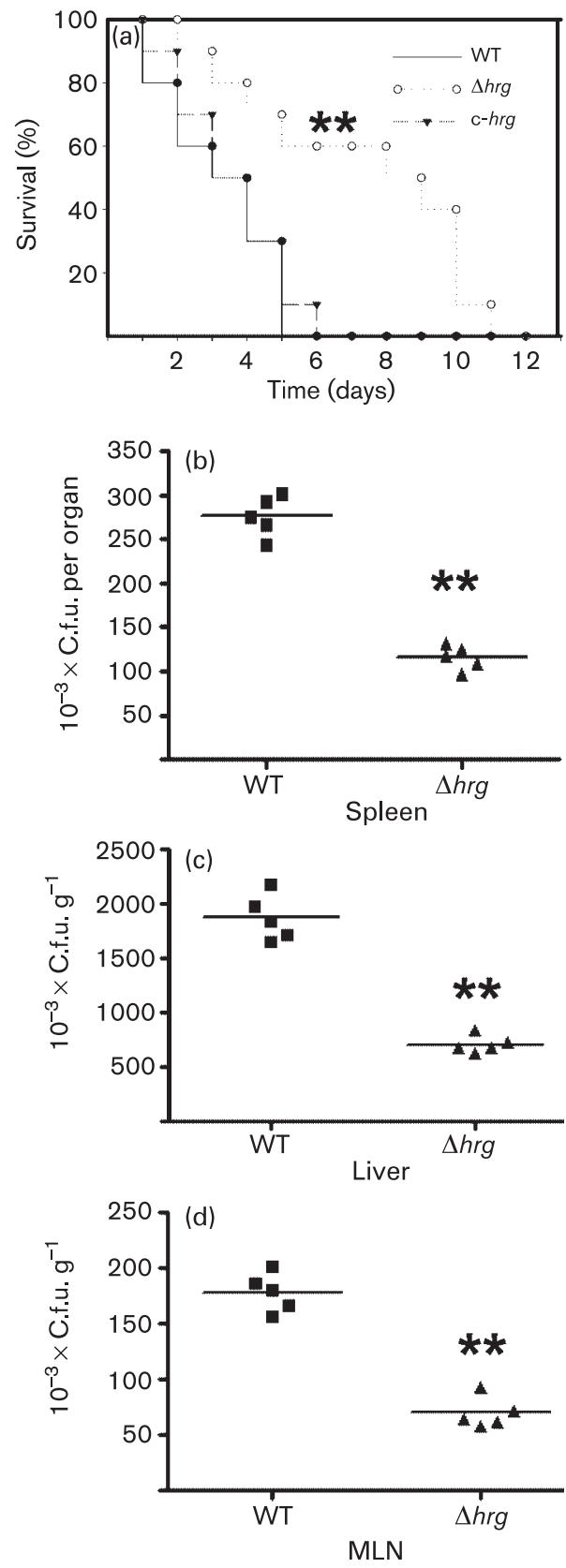

Fig. 6. Mouse model of infection. (a) Groups of 10 mice were inoculated with $10^{7}$ c.f.u. of the WT, $\Delta h r g$ or c- $h r g$ strain and monitored for survival for 12 days. (b-d) Bacterial load after 5 days of infection in spleen (b), liver (c) and mesenteric lymph nodes (MLN) (d) of BALB/c mice infected orally with $10^{6}$ bacteria per mouse. Mice were sacrificed on the fifth day after inoculation and the homogenized organs were plated to count c.f.u. Plots are representative of three independent experiments. ${ }^{\star \star} P<0.01$ (Mann-Whitney $U$ test).

before infection, which abrogates ROS production, reversed the growth defect of the $\Delta h r g$. We obtained similar result with a pharmacological inhibitor of NADPH oxidase, PAO (Le Cabec \& Maridonneau-Parini, 1995). So
Table 1. Competitive indices of mixed infections using WT, $\Delta h r g$ and c-hrg strains

The data shown are representative of three experiments with 10 mice in each group.

\begin{tabular}{|lcccc|}
\hline Strain 1 & Strain 2 & \multicolumn{2}{c|}{$\begin{array}{c}\text { Median competitive index of strain 2 } \\
\text { with respect to strain 1 }\end{array}$} \\
\cline { 3 - 5 } & & Spleen & Liver & MLN\$ \\
\hline $\mathrm{WT}^{*}$ & $\mathrm{WT} \dagger$ & 1.17 & 1.18 & 1.20 \\
$\mathrm{WT}^{\star}$ & $\Delta h r g$ & $0.36 \ddagger$ & $0.32 \ddagger$ & $0.40 \ddagger$ \\
$\mathrm{WT}^{\star}$ & $\mathrm{c}-\mathrm{hrg}$ & 1.30 & 1.35 & 1 \\
\hline
\end{tabular}

${ }^{\star}$ Resistant to carbenicillin.

$\dagger$ Resistant to nalidixic acid, and the parent strain for all knockout strains. The two WT strains were isogenic.

$\ddagger P<0.05$ (Mann-Whitney $U$ test).

§Mesenteric lymph nodes.

it can be predicted that either by quenching the ROS produced or by inhibiting the NADPH oxidase function the intracellular growth of the $\Delta h r g$ strain can be restored to the WT level. The increased susceptibility of the $\Delta h r g$ strain to $\mathrm{H}_{2} \mathrm{O}_{2}$ in vitro suggests that the genes necessary to counteract $\mathrm{H}_{2} \mathrm{O}_{2}$ are probably non-functional in this strain. Further, our microarray data are in accordance with the hypothesis that under $\mathrm{H}_{2} \mathrm{O}_{2}$ stress a reduced level of catalase in the $\Delta \mathrm{hrg}$ strain makes the strain much more susceptible than the WT to $\mathrm{H}_{2} \mathrm{O}_{2}$-mediated killing. DNA repair after oxidative damage is a survival strategy for the pathogen. UvrA and DNA polymerase III are required to repair DNA damage by $\mathrm{H}_{2} \mathrm{O}_{2}$ (Farr \& Kogoma, 1991). The decreased expression of both uvrA and DNA polymerase III, theta subunit (HolE) in the $\Delta h r g$ strain upon $\mathrm{H}_{2} \mathrm{O}_{2}$ treatment might also be a reason for the observed hypersensitive phenotype. The specificity of the resistance to $\mathrm{H}_{2} \mathrm{O}_{2}$ makes the $\mathrm{hrg}$ gene unique. The Hrg-overproducing strain showed an increased survival advantage over the WT strain upon $\mathrm{H}_{2} \mathrm{O}_{2}$ stress. Virulent Salmonella is known to inhibit the fusion of the Salmonella-containing vacuole with NADPH oxidase, thereby leading to increased bacterial survival in the macrophages (Vazquez-Torres et

Table 2. Downregulated genes in the $\Delta h r g$ strain upon oxidative stress

\begin{tabular}{|lcc|}
\hline Gene name/product & $\begin{array}{c}\text { Fold } \\
\text { decrease }\end{array}$ & P value \\
\hline Catalase hydroperoxidase HPI (I) $(k a t G)$ & 0.53 & 0.0029 \\
Acid shock protein & 0.44 & 0.0048 \\
NADH dehydrogenase I chain G $(n u o G)$ & 0.47 & 0.0003 \\
NADH dehydrogenase I chain N (nuoN) & 0.40 & 0.0007 \\
uvrA & 0.40 & 0.004 \\
DNA polymerase III theta subunit (holE) & 0.33 & 0.0044 \\
\hline
\end{tabular}


al., 2000). The increased co-localization of gp91phox with the $\Delta h r g$ strain indicates more active assembly of the phagocyte oxidase system near the bacterial site when compared to the WT strain and thus we can speculate that Hrg-regulated genes might be directly or indirectly involved in inhibiting gp91phox co-localization to the SCV.

\section{Concluding remarks}

There are several Salmonella genes that are required for resistance to oxidative stress, such as katE, katG, sod, rpoS and slyA (Papp-Szabò et al., 1994; Sly et al., 2002; RobbeSaule et al., 2001; Buchmeier et al., 1997). In vitro hypersensitivity of the $\Delta h r g$ strain to $\mathrm{H}_{2} \mathrm{O}_{2}$ and the high level of ROS produced by macrophages in response to $\Delta \mathrm{hrg}$ infection suggest a role for Hrg-regulated genes in quenching the $\mathrm{H}_{2} \mathrm{O}_{2}$ both in vitro and in vivo. The increased survival of the Hrg-overexpressing strain upon oxidative stress also supports this hypothesis. The Hrg-regulated genes also appear to prevent the NADPH phagocytic oxidase trafficking towards the Salmonella-containing vacuoles. Hence, our data suggest that STM0952 ( $\mathrm{hrg})$ transcriptionally regulates multiple gene loci involved in the pathogenesis of $S$. Typhimurium, and the regulation might vary in vitro and in vivo depending on the requirement of efficient infection. Our microarray results showed that catalase hydroperoxidase HPI ( $k a t G$ gene), uvrA and DNA polymerase III theta subunit (holE gene) were downregulated significantly in the knockout strain. This result would explain the enhanced ROS production and $\mathrm{H}_{2} \mathrm{O}_{2}$ hypersensitivity of the $\Delta \mathrm{hrg}$ strain, in which $\mathrm{H}_{2} \mathrm{O}_{2}$ could not be effectively quenched and DNA repair is hindered. Although OxyR is known to positively regulate catalase expression, we propose that $\mathrm{Hrg}$ might function in conjunction with OxyR in this regulation. Thus, this study provides a basis for further work to identify the exact mechanism of the regulation by $h r g$ on its downstream genes, which will provide important insights into oxygen-dependent antimicrobial mechanisms in Salmonella pathogenesis.

\section{ACKNOWLEDGEMENTS}

This work was supported by a grant, Provision (2A) Tenth Plan (191/ MCB), from the Director of Indian Institute of Science, ICMR Centre for Medical Microbiology and DBT programme support on Basic Biology of Microbial Pathogens. We would like to thank Dr Omana Joy for helping us with the FACS analysis and Kiran Kumar Panguluri for construction of the STM1625 mutant. We thank Dr Balaji and Dr Nandakumar for their critical suggestions. We would also like to thank Genotypic Technologies, Agilent Technologies for the microarray experiment.

\section{REFERENCES}

Beuzon, C. R. \& Holden, D. W. (2001). Use of mixed infections with Salmonella strains to study virulence genes and their interactions in vivo. Microbes Infect 3, 1345-1352.
Buchmeier, N., Bossie, S., Chen, C. Y., Fang, F. C., Guiney, D. G. \& Libby, S. J. (1997). SlyA, a transcriptional regulator of Salmonella typhimurium, is required for resistance to oxidative stress and is expressed in the intracellular environment of macrophages. Infect Immun 65, 3725-3730.

Cadenas, E. (1989). Biochemistry of oxygen toxicity. Annu Rev Biochem 58, 79-110.

Chakravortty, D., Hansen-Wester, I. \& Hensel, M. (2002). Salmonella pathogenicity island 2 mediates protection of intracellular Salmonella from reactive nitrogen intermediates. J Exp Med 195, 1155-1166.

Christman, M. F., Morgan, R. W., Jacobson, F. S. \& Ames, B. N. (1985). Positive control of a regulon for defenses against oxidative stress and some heat shock proteins in Salmonella typhimurium. Cell 41, 753-762.

Christman, M. F., Storz, G. \& Ames, B. N. (1989). OxyR, a positive regulator of hydrogen peroxide-inducible genes in Escherichia coli and Salmonella typhimurium, is homologous to a family of bacterial regulatory proteins. Proc Natl Acad Sci U S A 86, 3484-3488.

Datsenko, K. A. \& Wanner, B. L. (2000). One-step inactivation of chromosomal genes in Escherichia coli K-12 using PCR products. Proc Natl Acad Sci U S A 97, 6640-6645.

De Groote, M. A., Ochsner, U. A., Shiloh, M. U., Nathan, C., McCord, J. M., Dinauer, M. C., Libby, S. J., Vasquez-Torres, A., Xu, Y. \& Fang, F. C. (1997). Periplasmic superoxide dismutase protects Salmonella from products of phagocyte NADPH-oxidase and nitric oxide synthase. Proc Natl Acad Sci U S A 94, 13997-14001.

Eriksson, S., Lucchini, S., Thompson, A., Rhen, M. \& Hinton, J. C. D. (2003). Unravelling the biology of macrophage infection by gene expression profiling of intracellular Salmonella enterica. Mol Microbiol 47, 103-118.

Farr, S. B. \& Kogoma, T. (1991). Oxidative stress response in Escherichia coli and Salmonella typhimurium. Microbiol Rev 55, 561-585.

Foster, N., Hulme, S. D. \& Barrow, P. A. (2003). Induction of antimicrobial pathways during early-phase immune response to Salmonella spp. in murine macrophages: gamma interferon (IFN- $\gamma$ ) and upregulation of IFN- $\gamma$ receptor alpha expression are required for $\mathrm{NADPH}$ phagocytic oxidase gp91-stimulated oxidative burst and control of virulent Salmonella spp. Infect Immun 71, 4733-4741.

Garcia Vescovi, E., Soncini, F. C. \& Groisman, E. A. (1994). The role of the PhoP/PhoQ regulon in Salmonella virulence. Res Microbiol 145, 473-480.

Hensel, M., Shea, J. E., Gleeson, C., Jones, M. D., Dalton, E. \& Holden, D. W. (1995). Simultaneous identification of bacterial virulence genes by negative selection. Science 269, 400-403.

Kredich, N. M. (1971). Regulation of L-cysteine biosynthesis in Salmonella typhimurium. I. effects of growth on varying sulfur sources and O-acetyl-L-serine on gene expression. J Biol Chem 246, 3474-3484.

Le Cabec, V. \& Maridonneau-Parini, I. (1995). Complete and reversible inhibition of NADPH oxidase in human neutrophils by phenylarsine oxide at a step distal to membrane translocation of the enzyme subunits. J Biol Chem 270, 2067-2073.

Leusen, J. H., deBoer, W. M., Bolscher, B. G. J. M., Hilaries, P. M., Weening, R. S., Ochs, H. J., Roos, D. \& Verhoeven, A. J. (1994). A point mutation in gp91 $1^{\text {phox }}$ of cytochrome $b_{558}$ of human NADPH oxidase leading to defective translocation of the cytosolic proteins p47-phox and p67-phox. J Clin Invest 93, 2120-2126.

Libby, S. J., Adams, L. G., Ficht, T. A., Allen, C., Whitford, H. A., Buchmeier, N. A., Bossie, S. \& Guiney, D. G. (1997). The spv genes on the Salmonella dublin virulence plasmid are required for severe 
enteritis and systemic infection in the natural host. Infect Immun 65, $1786-1792$

Libby, S. J., Lesnick, M., Hasegawa, P., Weidenhammer, E. \& Guiney, D. G. (2000). The Salmonella virulence plasmid spv genes are required for cytopathology in human monocyte-derived macrophages. Cell Microbiol 2, 49-58.

Libby, S. J., Lesnick, M., Hasegawa, P., Kurth, M., Belcher, C., Fierer, J. \& Guiney, D. G. (2002). Characterization of the spv locus in Salmonella enterica serovar Arizona. Infect Immun 70, 3290-3294.

Mastroeni, P., Vazquez-Torres, A., Fang, F. C., Xu, Y., Khan, S., Hormaeche, C. E. \& Dougan, G. (2000). Antimicrobial actions of the NADPH phagocyte oxidase and inducible nitric oxide synthase in experimental salmonellosis. II. Effects on microbial proliferation and host survival in vivo. J Exp Med 192, 237-248.

Miller, R. A. \& Britigan, B. E. (1997). Role of oxidants in microbial pathophysiology. Clin Microbiol Rev 10, 1-18.

Miller, S. I., Kukral, A. M. \& Mekalanos, J. J. (1989). A two-component regulatory system ( $p h o P$ phoQ) controls Salmonella typhimurium virulence. Proc Natl Acad Sci U S A 86, 5054-5058.

Mouy, R., Fischer, A., Vilmer, E., Seger, R. \& Griscelli, C. (1989). Incidence, severity, and prevention of infections in chronic granulomatous disease. J Pediatr 114, 555-560.

Ochsner, U. A., Vasil, M. L., Alsabbagh, E., Parvatiyar, K. \& Hassett, D. J. (2000). Role of the Pseudomonas aeruginosa oxyR-recG operon in oxidative stress defense and DNA repair: OxyR-dependent regulation of katB-ankB, ahpB, and ahpC-ahp. J Bacteriol 182, 4533-4544.

Pagán-Ramos, E., Song, J., McFalone, M., Mudd, M. H. \& Deretic, V. (1998). Oxidative stress response and characterization of the oxyRahpC and furA-katG loci in Mycobacterium marinum. J Bacteriol 180, 4856-4864.

Papp-Szabò, E., Firtel, M. \& Josephy, P. D. (1994). Comparison of the sensitivities of Salmonella typhimurium oxyR and kat $G$ mutants to killing by human neutrophils. Infect Immun 62, 2662-2668.

Robbe-Saule, V., Coynault, C., Ibanez-Ruiz, M., Hermant, D. \& Norel, F. (2001). Identification of a non-haem catalase in Salmonella and its regulation by $\operatorname{RpoS}\left(\sigma^{\mathrm{S}}\right)$. Mol Microbiol 39, 1533-1545.

Rosenberger, C. M. \& Finlay, B. B. (2002). Macrophages inhibit Salmonella Typhimurium replication through MEK/ERK kinase and phagocyte NADPH oxidase activities. J Biol Chem 277, 18753-18762.

Russell, D. A., Byrne, G. A., O'Connell, E. P., Boland, C. A. \& Meijer, W. G. (2004). The LysR-type transcriptional regulator VirR is required for expression of the virulence gene vapA of Rhodococcus equi ATCC 33701. J Bacteriol 186, 5576-5584.

Sambrook, J., Fritsch, E. F. \& Maniatis, T. (1989). Molecular Cloning: a Laboratory Manual, 2nd edn. Cold Spring Harbor, NY: Cold Spring Harbor Laboratory.
Schell, M. A. (1993). Molecular biology of the LysR family of transcriptional regulators. Annu Rev Microbiol 47, 597-626.

Sly, L. M., Guiney, D. G. \& Reiner, N. E. (2002). Salmonella enterica serovar Typhimurium periplasmic superoxide dismutases SodCI and SodCII are required for protection against the phagocyte oxidative burst. Infect Immun 70, 5312-5315.

Sun, Y. H., den Hartigh, A. B., de Lima Santos, R., Adams, L. G. \& Tsolis, R. M. (2002). virB-mediated survival of Brucella abortus in mice and macrophages is independent of a functional inducible nitric oxide synthase or NADPH oxidase in macrophages. Infect Immun 70, $4826-4832$.

Taylor, P. D., Inchley, C. J. \& Gallagher, M. P. (1998). The Salmonella typhimurium AhpC polypeptide is not essential for virulence in $\mathrm{BALB} / \mathrm{c}$ mice but is recognized as an antigen during infection. Infect Immun 66, 3208-3217.

Tseng, H. J., McEwan, A. G., Apicella, M. A. \& Jennings, M. P. (2003). OxyR acts as a repressor of catalase expression in Neisseria gonorrhoeae. Infect Immun 71, 550-556.

Vazquez-Torres, A., Xu, Y., Jones-Carson, J., Holden, D. W., Lucia, S. M., Dinauer, M. C., Mastroeni, P. \& Fang, F. C. (2000). Salmonella pathogenicity island 2-dependent evasion of the phagocyte NADPH oxidase. Science 287, 1655-1658.

Von Knethen, A. A. \& Brüne, B. (2001). Delayed activation of PPAR by LPS and IFN $\gamma$ attenuates the oxidative burst in macrophages. FASEB J 15, 535-544.

Wautier, M. P., Chappey, O., Corda, S., Stern, D. M., Schmidt, A. M. \& Wautie, J. L. (2001). Activation of NADPH oxidase by AGE links oxidant stress to altered gene expression via RAGE. Am J Physiol Endocrinol Metab 280, E685-E694.

Wilson, C. B., Tsai, V. \& Remington, J. S. (1980). Failure to trigger the oxidative metabolic burst by normal macrophages: possible mechanism for survival of intracellular pathogens. J Exp Med 151, 328-346.

Wojtaszek, P. (1997). Oxidative burst: an early plant response to pathogen infection. Biochem J 322, 681-692.

Wu, J. \& Weiss, B. (1991). Two divergently transcribed genes, soxR and soxS, control a superoxide response regulon of Escherichia coli. J Bacteriol 173, 2864-2871.

Xiao, L., Pimentel, D. R., Wang, J., Singh, K., Colucci, W. S. \& Sawyer, D. B. (2002). Role of reactive oxygen species and $\mathrm{NAD}(\mathrm{P}) \mathrm{H}$ oxidase in $\alpha_{1}$-adrenoceptor signaling in adult rat cardiac myocytes. Am J Physiol Cell Physiol 282, C926-C934.

Ygberg, S. E., Clements, M. O., Rytkonen, A., Thompson, A., Holden, D. W., Hinton, J. C. \& Rhen, M. (2006). Polynucleotide phosphorylase negatively controls $s p v$ virulence gene expression in Salmonella enterica. Infect Immun 74, 1243-1254.

Edited by: P. H. Everest 